\title{
Exact Similarity Solution for Unsteady Isothermal Flow Behind a Shock Wave in Self- gravitating Non-ideal Gas
}

\author{
G. Nath
}

Department of Mathematics, Motilal Nehru National Institute of Technology Allahabad, Allahabad 211004, India

Corresponding Author Email: gnath@mnnit.ac.in; gn_chaurasia_univgkp@yahoo.in

https://doi.org/10.18280/mmc_b.882-404

Received: 14 March 2019

Accepted: 22 August 2019

\section{Keywords:}

exact similarity solution, gravitating medium, shock waves, non-ideal gas, isothermal flows

\begin{abstract}
The propagation of strong spherical shock wave in non-ideal gas under the influence of gravitational field is discussed. The total energy of the shock wave increases with time. An exact similarity solution is obtained in the case when the medium is non-ideal under the influence of a central mass $(m)$. The effects of variation of the parameter of nonidealness of the gas and the ratio of the specific heats of the gas are workout in detail. It is shown that the shock strength and the isothermal compressibility decrease with an increase in the value of the ratio of specific heats of the gas or the parameter of nonidealness of the gas. Also, it is obtained that an increase in the value of the parameter of non-idealness of the gas and the ratio of the specific heats has same effect on the flow variables and on the shock strength.
\end{abstract}

\section{INTRODUCTION}

Due to sudden released of a large amount of energy in a small region, for example an explosion, a disturbance headed by a strong shock wave called the blast wave is produced and propagates into the surround medium. The mathematical formulation for this phenomena is studied in [1-2] in the case of adiabatic fluid dynamics with Rankine-Hugoniot conditions which comes from the conservation of mass, momentum and energy through the shock front. The jump conditions for strong shock depend on the shock velocity and the density ahead of the front only. For the strong shock, some authors have constracted exact solutions of the blast wave in a sphereical geometry because the study of spherical symmetry motion is important for the theory of explosion in various medium, for example air and water (see [1, 3, 4-7]).

Carrus et al. [6] studied the propagation of shock waves in a gas under the gravitational attraction of a central body of the fixed mass (Roche model) and obtained similarity solutions by using numerical method. The method for obtaining analytical solution of the same problem was discussed by Rogers [5]. The formation of self-similar problems and examples describing the adiabatic motion of gas models of star are considered in [1, 8-10].

In extreme conditions that prevail in most of the problems associated with shock waves, the assumption that the gas is ideal is no longer valid. In recent years, several studies have been performed concerning the problem of shock waves in non-ideal gases (see [11-17] among others). In all of the works mentioned above, the influence of gravitational field under the isothermal flow condition is not considered by any of the author to obtain the exact similarity solution. The gravitational force has considerable effect on many astrophysical problems. Also, we have considered the gas to be non-ideal. The popular alternative to the ideal gas is a simplified van der Waals model. In the present work, we too adopt this as our model of a nonideal gas to extend the problem treated by Vishwakarma et al.
[18] and to obtain the self-similar solutions for unsteady isothermal flow behind a strong spherical shock wave propagating in a non-ideal gas under the influence of gravitational field. In order to obtain exact similarity solutions, the initial density of the medium is taken to be constant. The exact similarity solutions are obtained in the case of isothermal flow with the strong shock conditions by using the 'Product Solutions' of McVittie [19] to evaluate the flow variables distribution behind the shock front. The exact solution using 'Product Solutions' of McVittie [19] in the case of adiabatic flow with or without gravitation are also, studied in [20-24]. The assumption of isothermal flow is physically realistic, when radiation heat transfer effects are implicitly present. As the shock propagates, the temperature behind it increases and becomes very large so that there is intense transfer of energy by radiation. This causes the temperature gradient to approach zero; that is, the dependent temperature tends to become uniform behind the shock front, and the flow becomes isothermal [25-28]. With this assumption, we obtain the exact solution in Section 4. The effects of various parameters on the shock velocity, shock strength and on the flow variables are shown in Figures 1-2 and Table 1 and discussed in Section 5.

The effects of variation of the parameter of non-idealness of the gas and the ratio of the specific heat of the gas on the shock strength, isothermal compressibility and on the flow variables are investigated. It is found that the shock strength and isothermal compressibility decrease with an increase in the ratio of the specific heat of the gas or the parameter of nonidealness of the gas. Also, it is found that an increase in the value of the parameter of non-idealness of the gas and the adiabatic exponent have similar effect on the flow variables and on the shock strength.

\section{BASIC EQUATIONS OF MOTIONS}

The fundamental equations governing the one-dimensional, 
unsteady isothermal and spherically symmetric motion of a non-ideal gas under the influence of a gravitational field may be written as $([6-7,18,25])$.

$$
\begin{gathered}
\frac{\partial \rho}{\partial t}+u \frac{\partial \rho}{\partial r}+\rho \frac{\partial u}{\partial r}+\frac{2 u \rho}{r}=0 \\
\frac{\partial u}{\partial t}+u \frac{\partial u}{\partial r}+\frac{1}{\rho} \frac{\partial p}{\partial r}+\frac{G m}{r^{2}}=0 \\
\frac{\partial T}{\partial r}=0
\end{gathered}
$$

where, $r$ and $t$ are the independent space and time coordinates, $\mathrm{u}$ is the fluid velocity, $\rho$ is the density, $\mathrm{p}$ is the pressure, $\mathrm{T}$ is the temperature, $m$ is the central mass at the origin, and $G$ is the gravitational constant.

The assumption that the gas is ideal is no longer valid when the flow takes place at higher temperature [16]. To obtain the deviation in solution from the case of ideal gas in the non-ideal gas, we adopt a simple model. We assume that the gas obey a simplified van der Waal equation of state of form (Wu and Roberts [13], Roberts and Wu [14], Nath [15])

$$
p=\frac{R \rho T}{1-b \rho}, E=C_{v} T=\frac{p(1-b \rho)}{\rho(\gamma-1)},
$$

where, $\mathrm{R}$ is the gas constant, $\gamma$ is the ratio of specific heats, $\mathrm{E}$ is the internal energy per unit mass of the gas, $C_{v}=\frac{R}{\gamma-1}$ is the specific heat at constant volume, constant $b$ is the van der Waal excluded volume, it places a limit, $\rho{\frac{1}{b_{\text {max }}}}$, on the density of the gas.

For the self-similar solution (Sedov [1]) the relation the shock velocity $W_{s}=\frac{d r_{s}}{d t}$ is assumed to vary as $([18-24])$

$$
W_{s}^{2}=A^{2} r_{s}^{-\alpha}
$$

where, $r_{s}$ is the shock radius, $\mathrm{A}$ and $\alpha$ are constant

A strong spherical shock wave is supposed to be propagating in the undisturbed medium under the influence of gravitational field. The flow variables immediately ahead of shock front are

$$
u_{a}=0, \rho=\rho_{a}=\text { constant }
$$

where, subscript ' $a$ ' refers to the conditions immediately ahead of the shock front.

\section{BOUNDARY CONDITIONS}

As the pressure ahead of a strong shock is very small in comparison to the pressure behind of the shock, and therefore it is neglected (Zel'dovich and Raizer [8], Vishwakarma and Nath [16]) i.e. $p_{a} \approx 0, E_{a} \approx 0$. Therefore, the Rankine-Hugoniot jump conditions across the strong shock wave propagating in a non-ideal gas are given by the principal of conservation of mass, momentum and energy, namely,

$$
\rho_{n}=\frac{\rho_{a}}{\beta}, u_{n}=(1-\beta) W_{s}, p_{n}=(1-\beta) \rho_{a} W_{s}^{2},
$$

where, the subscript $\mathrm{n}$ denotes the condition immediately behind the shock front, the density ratio $\beta(0<\beta<1)$ across the shock front is obtained by the relation

$$
\beta=\frac{(2 \bar{b}+\gamma-2)}{(\gamma+1)},
$$

where, $\left(F_{n}-F_{a}\right)$ is neglected in comparison with the product of $p_{n}$ and $W_{S}$ as the shock is strong (Laumbach and Probstein [27], Nath [15, 25], Vishwakarma and Nath [16]) to derive equation (8) above, F is the radiation flux and $\bar{b}=\boldsymbol{b} \boldsymbol{\rho}_{\boldsymbol{a}}$.

Eq. (4) together with (5) gives

$$
\frac{p}{p_{n}}=\frac{\rho\left(1-b \rho_{n}\right)}{\rho_{n}(1-b \rho)}
$$

For an isentropic change of state of the non-ideal gas, we may calculate the so-called speed of sound in non-ideal gas for a given internal volume of the gas $b$ as follows,

$$
a_{\text {sound }}=\left(\frac{d \mathrm{p}}{d \rho}\right)_{\mathrm{S}}^{\frac{1}{2}}=\left[\frac{\gamma p}{\rho(1-b \rho)}\right]^{\frac{1}{2}}
$$

where, the subscript ' $S$ ' refers to the process of constant entropy.

The isothermal compressibility of non-ideal gas may be calculated as (Nath [15]).

$$
C_{i s o t h}=\frac{1}{\rho a_{i s o t h}^{2}}
$$

where, $\quad a_{\text {isot } h}=\left(\frac{d p}{d \rho}\right)_{T}^{\frac{1}{2}}=\left[\frac{p}{(1-b \rho) \rho}\right]^{\frac{1}{2}} \quad$ expressing the isothermal sound speed and the subscript ' $\mathrm{T}$ ' refers to the process of constant temperature.

\section{SELF-SIMILARITY TRANSFORMATIONS}

To obtain the similarity solution. We write the unknown variables in the following form (Nath et al. [21], Vishwakarma et al. [18]).

$$
u=V(x) W_{s}, \rho=\rho_{a} D(x), p=\rho_{a} W_{x}^{2} P(x)
$$

where, $\mathrm{V}(\mathrm{x}), \mathrm{D}(\mathrm{x})$, and $\mathrm{P}(\mathrm{X})$ are the function of $\mathrm{x}$ only, $\mathrm{x}=\mathrm{r} / \mathrm{r}_{\mathrm{s}}$ is the similarity variable (dimensionless variable).

Eq. (9) with the aid of the Eqns. (7) and (12) yields a relation between $\mathrm{P}(\mathrm{x})$ and $\mathrm{D}(\mathrm{x})$ in the form

$$
P(x)=\frac{D(x)(1-\beta)(\beta-\bar{b})}{(1-\bar{b} D(x))}
$$

Using equation (13) and similarity transformations (12), equations (1) and (2) can be transformed and simplified to 


$$
\begin{gathered}
(V-x) \frac{d D}{d x}+D \frac{d V}{d x}+\frac{2 D V}{x}=0 \\
(V-x) \frac{d V}{d x}-\frac{\alpha V}{2}+\frac{1}{D} \frac{(1-\beta)(\beta-\bar{b})}{(\beta-\bar{b} D)^{2}} \frac{d D}{d x}+\frac{G_{0}}{x^{2}}=0
\end{gathered}
$$

where, $G_{0}=\left(\frac{G m}{A^{2}}\right)$, and it is necessary to use $\alpha=1$, to obtain the similarity solution.

Using the similarity transformations (7), the shock conditions (7), transformed into

$$
V(1)=(1-\beta), D(1)=\frac{1}{\beta}, P(1)=(1-\beta) .
$$

We assume the product solution of the "progressive wave" in the form (c.f. Mc. Vittie [14])

$$
\begin{gathered}
u=\frac{a(t)}{t} r, \\
\rho=(\lambda+1) f(t) t^{-2 \tau} \eta^{\lambda-2}, \\
p=\tau^{2} f(t) t^{-2} b(t) \eta^{\lambda-2},
\end{gathered}
$$

where, $\eta=\mathrm{rt}^{-\tau}, \lambda$ and $\tau$ are constants, and $\mathrm{a}, \mathrm{f}$ and $\mathrm{b}$ are functions of $t$ that satisfy the following equation

$$
\begin{gathered}
a(t)=\frac{\tau \lambda-t f^{\prime} / f}{\lambda+1} \\
(\lambda-2) b(t) \tau^{2}=\eta^{2}(\lambda+1)\left[\left(a(t)-a^{2}(t)-t a^{\prime}(t)\right)-\frac{G m t^{2-3 \tau}}{\eta^{3}}\right]
\end{gathered}
$$

It can be easily seen that these equations satisfy the Eqns. (1) and (2) identically. After converting this solution to a similarity one, it follows that 'a' should be a constant $a=\frac{2(1-\beta)}{(\alpha+2)}$, we apply the boundary conditions (16) in (17) and (18), we obtain the solution as

$$
\begin{aligned}
& W(x)=(1-\beta) x, \\
& D(x)=\frac{1}{\beta} x^{\lambda-2},
\end{aligned}
$$

These solutions (22) and (23) satisfy differential equations (14) and (15) identically, and therefore they constitute a solution of these differential equations (13) and (14). From equations (12) and (24),

$$
P(x)=\frac{(1-\beta)(\beta-\bar{b}) x^{\lambda-2}}{\left(\beta-\bar{b} x^{\lambda-2}\right)}
$$

Now, substituting equations (22)-(24) into equations (13)(15), we obtain

$$
\begin{gathered}
\lambda=\left(\frac{3}{\beta}-1\right), \\
G_{0}=\frac{(1-\beta) x\left[x^{2}(2 \beta+\alpha)\left(\beta-\bar{b} x^{\lambda-2}\right)^{2}-2 \beta^{2}(\beta-\bar{b})(\lambda-2)\right]}{2\left(\beta-\bar{b} x^{\lambda-2}\right)^{2}}
\end{gathered}
$$

The total energy $\mathrm{E}_{\text {Total }}$ of the flow field behind the spherical shock front is given by

$$
E_{\text {Total }}=4 \pi \int_{0}^{r_{S}}\left[\frac{p(1-b \rho)}{(\gamma-1) \rho}+\frac{1}{2} \mathrm{u}^{2}-\frac{G m}{r}\right] \rho r^{2} d r
$$

Using Eq. (12) the above Eq. (27) transformed into

$$
E_{\text {Total }}=4 \pi \rho_{a} A^{2} r_{s}^{3-\alpha} \int_{0}^{1}\left[\frac{P(1-\bar{b} D)}{(\gamma-1)}+\frac{1}{2}\left(V^{2} D\right)-\frac{D G_{0}}{x}\right] x^{2} d x .
$$

This Eq. (28) shows that the total energy of the disturbance is not constant and varies as $r_{s}^{3-\alpha}$. Eqns. (22)-(24) give the solution of our considered problem. The solution we have obtained is an example of exact solution in a non-ideal gas with constant density in the case of isothermal flow under the influence of a gravitational field and similar to exact solutions obtained in ordinary gas dynamics by Mc.Vittie [19] in the case of adiabatic flow, and in magnetogasdynamics with radiative heat flux by Vishwakarma et al. [18]. The exact solutions using Mc.Vittie [19], method in the case of isothermal flow in non-ideal gas under the influence of gravitational field is reported first time.

Using equations (12) in (11), we obtain the expression for the isothermal compressibility as

$$
\left(C_{\text {isoth }}\right) \rho_{a} W_{s}^{2}=\frac{(1-\bar{b} D)}{P(x)}
$$

\section{RESULTS AND DISCUSSION}

Table 1. Problem parameter values for different values of $\gamma$ and $\bar{b}$

\begin{tabular}{cccc}
\hline$\gamma$ & $\overline{\boldsymbol{b}}$ & $\boldsymbol{\beta}$ & $\boldsymbol{\lambda}=\left(\frac{\mathbf{3}}{\boldsymbol{\beta}}-\mathbf{1}\right)$ \\
\hline $4 / 3$ & 0 & 0.14285 & 20 \\
& 0.05 & 0.18571 & 15.1538 \\
& 0.1 & 0.22857 & 12.1250 \\
$5 / 3$ & 0 & 0.25000 & 11 \\
& 0.05 & 0.28750 & 9.43478 \\
& 0.1 & 0.32500 & 8.23077 \\
\hline
\end{tabular}

For density and pressure to remain finite at the point of symmetry the inequality obtained from (23). i.e. $\lambda>2$ should hold. To obtain the solution the values of the physical parameters are taken as (see Refs. [15,22-23]): $\gamma=4 / 3,5 / 3$ $\bar{b}=0,0.05,0.1$. The obtained solutions are shown in Figures 1 and 2 and Table 1. Figures $1(\mathrm{a}-\mathrm{c})$ and $2(\mathrm{a}, \mathrm{b})$ show that the fluid velocity $\mathrm{U}(\mathrm{x})$, density $\mathrm{D}(\mathrm{x})$ and pressure $\mathrm{P}(\mathrm{x})$ decrease as we move from shock front to the point of symmetry. These flow variables are minimum at the point of symmetry and maximum at the shock. The isothermal compressibility 
$\left(C_{i s o t h}\right) \rho_{a} W_{s}^{2}$ increases and tends to positive infinity near the point of symmetry in general. The flow variables velocity, density and pressure vanish at the point of symmetry. Table 1 shows the values of the problem parameters for different values of $\gamma$ and $\bar{b}$.
The shock strength, fluid velocity $\mathrm{U}(\mathrm{x})$, and isothermal compressibility $\left(C_{\text {isoth }}\right) \rho_{a} W_{s}^{2}$ decrease; whereas the density $\mathrm{D}(\mathrm{x})$ and pressure $\mathrm{P}(\mathrm{x})$ increase with an increase in the parameter of non-idealness of the gas $\bar{b}$ or the specific heat ratio $\gamma$ (see Figures 1 and 2 and Table 1).

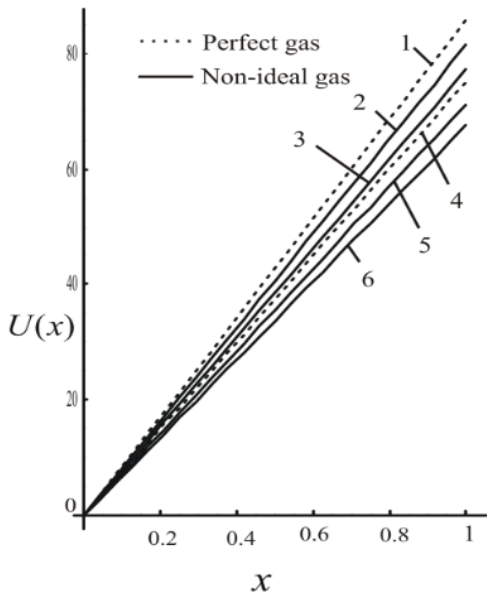

Figure 1(a)

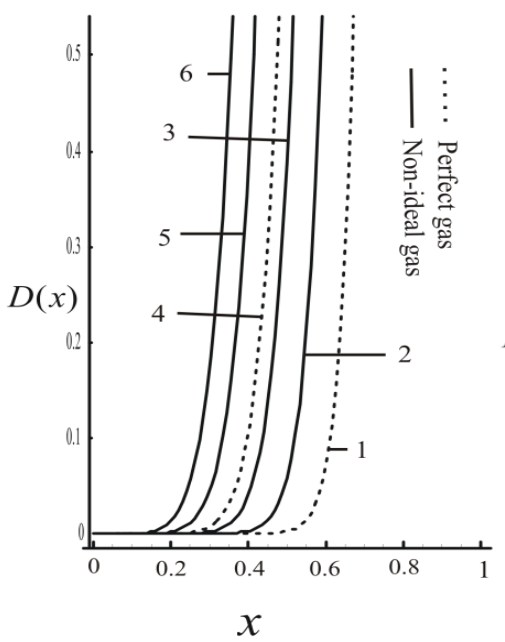

Figure 1(b)

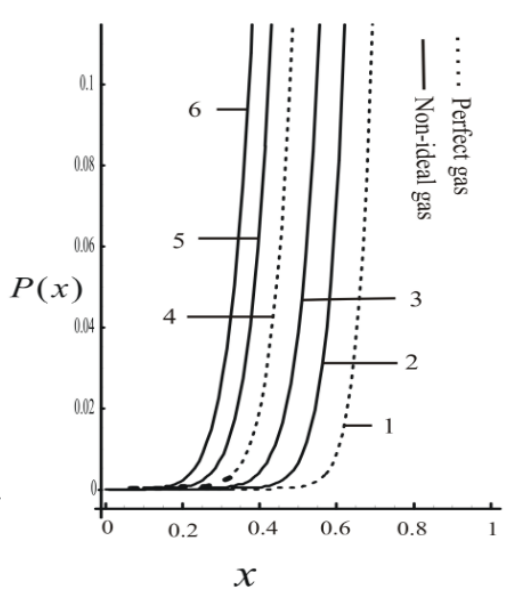

Figure 1(c)

Figure 1. Flow variables distribution in the flow field region behind the shock at $\gamma$ and $\bar{b}$ :

(a) fluid velocity $\mathrm{U}(\mathrm{x})$, (b) density $\mathrm{D}(\mathrm{x})$, (c) pressure $\mathrm{P}(\mathrm{x})$ :
1. $\gamma=4 / 3, \bar{b}=0$;
2. $\gamma=4 / 3, \bar{b}=0.05$
3. $\gamma=4 / 3, \bar{b}=0.1$
4. $\gamma=5 / 3, \bar{b}=0$;
5. $\gamma=5 / 3, \bar{b}=0.05$
6. $\gamma=5 / 3, \bar{b}=0.1$.
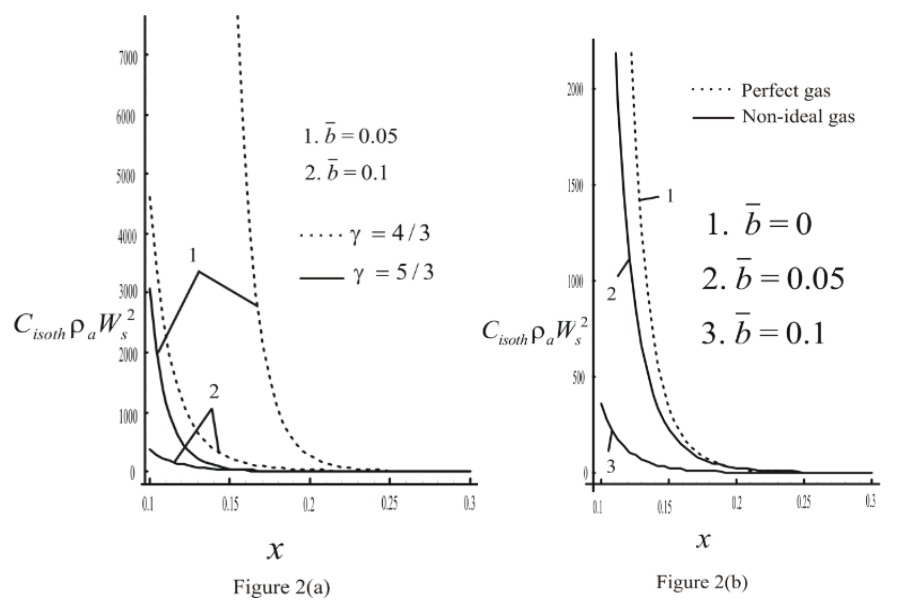

Figure 2. Variation of the isothermal compressibility $\left(C_{i s o t h}\right) \rho_{a} W_{s}^{2}$ :

(a) for different values of $\bar{b}$ and $\gamma$; (b) for different values of $\bar{b}$ with $\gamma=5 / 3$.

\section{CONCLUSION}

The similarity solutions for the propagation of strong spherical shock wave in a non-ideal gas under the influence of a gravitational field in the case of isothermal flow have been obtained. On the basis of the present study, we may draw the following conclusions:

An increase in $\bar{b}$ i.e. in the parameter of non-idealness of the gas decreases the shock strength, fluid velocity and isothermal compressibility. Moreover, it increases the density, pressure, and increase.

An increase in the value of the ratio of the gas specific heats has similar effects on the shock strength and on the flow variables.

The fluid velocity, density and pressure vanish at the point of symmetry.

\section{REFERENCES}

[1] Sedov, L.I. (1982). Similarity and Dimensional Methods in Mechanics. Mir Moscow.

[2] Murata, S. (2006). New exact solution of the blast wave problem in gas dynamics. Chaos, Solitons and Fractals, 28(2): 327-330. 
[3] Barenblatt, G.I. (2002). Scaling self-similarity and intermediate asymptotic. Dimensional Analysis and Intermediate Asymptotics, 14. Cambridge University Press.

[4] Taylor, J.L. (1955). An exact solution of spherical blast wave problem. The London, Edinburgh, and Dublin Philosophical Magazine and Journal of Science, 46(374): 317-320. https://doi.org/10.1080/14786440308521083

[5] Rogers, M.H. (1957). Analytic solutions for blast wave problem with an atmosphere of varying density. The Astrophysical Journal, 125: 478.

[6] Carrus, P., Fox, P., Hass, F., Kopal, Z. (1951). The propagation of shock waves in a stellar model with continuous density distribution. The Astrophysical Journal, 113: 496-518.

[7] Kopal, Z. (1954). The propagation of shock waves in self-gravitating gas sphere. The Astrophysical Journal, 120: 159-171.

[8] Zel'dovich, Y.B., Raizer, Y.P. (1967). Physics of Shock Waves and High Temperature Hydrodynamic Phenomena. Courier Corporation. Academic Press, New York.

[9] Summers, D. (1975). An idealized model of a magnetohydrodynamic spherical blast wave applied to a flare produced shock in the solar wind. Astronomy and Astrophysics, 45: 151-158.

[10] Lee, T.S., Chen, T. (1968). Hydromagnetic interplanetary shock waves. Planetary and Space Science, 16(12): 1483-1502. https://doi.org/10.1016/00320633(68)90061-5

[11] Anisimov, S.I., Spiner, O.M. (1972). Motion of an almost ideal gas in the presence of a strong point explosion. Journal of Applied Mathematics and Mechanics, 36(5): 883-887. https://doi.org/10.1016/0021-8928(72)90144$\mathrm{X}$

[12] Rao, M.P.R., Purohit, N.K. (1976). Self-similar problem in non-ideal gas. International Journal of Engineering Science, 14(1): 91-97. https://doi.org/10.1016/00207225(76)90059-8

[13] Wu, C.C., Roberts, P.H. (1993). Shock wave propagation in a sono-luminescing gas bubble. Physical review letters, 70(20): 3424-3427.

[14] Roberts, P.H., Wu, C.C. (1996). Structure and stability of a spherical implosion. Physics Letters A, 213(1-2): 5964. https://doi.org/10.1016/0375-9601(96)00082-5

[15] Nath, G. (2015). Similarity solutions for unsteady flow behind an exponential shock in an axisymmetric rotating non-ideal gas. Meccanica, 50(7): 1701-1715. https://doi.org/10.1007/s11012-015-0115-2

[16] Vishwakarma, J.P., Nath, G. (2007). Similarity solutions for the flow behind an exponential shock in a non-ideal gas. Meccanica, 42(4): 331-339. https://doi.org/10.1007/s11012-007-9058-6

[17] Vishwakarma, J.P., Nath, G. (2009). A self-similar solution of a shock propagation in a mixture of a nonideal gas and small solid particles. Meccanica, 44(3): 239-254. https://doi.org/10.1007/s11012-008-9166-y

[18] Vishwakarma, J.P., Srivastava, R.C., Kumar, A. (1987). An exact similarity solution in radiation magnetogasdynamics for the flows behind a spherical shock wave. Astrophysics and Space Science, 129(1): 45-52. https://doi.org/10.1007/BF00717856

[19] McVittie, G.C. (1953). Spherically symmetric solutions of the equations of gas dynamics. Proceedings of the
Royal Society of London. Series A. Mathematical and Physical Sciences, 220(1142): 339-355. https://doi.org/10.1098/rspa.1953.0191

[20] Srivastava, S.K., Singh, R.K. (1983). An exact similarity solution for a spherical shock wave in a self-gravitating system. Astrophysics and Space Science, 92(2): 365-372. https://doi.org/10.1007/BF00651301

[21] Nath, G., Dutta, M., Pathak, R.P. (2017). An exact solution for the propagation of shock waves in selfgravitating medium in the presence of magnetic field and radiative heat flux. AMSE Journals-AMSE IIETA publication-2017-Series: Modelling B, 86(4): 907-927. https://doi.org/10.18280/mmc_b.870404

[22] Vishwakarma, J.P., Patel, N. (2015). Magnetogasdynamic cylindrical shock waves in a rotating nonideal gas with radiation heat flux. Journal of Engineering Physics and Thermophysics, 88(2): 521-530. https://doi.org/10.1007/s10891-015-1217-3

[23] Nath, G., Singh, S., Srivastava, P. (2018). Exact solution for a magnetogasdynamical cylindrical shock wave in a self-gravitating rotating perfect gas with radiation heat flux and variable density. Journal of Engineering Physics and Thermophysics, 91: 1301-1312. https://doi.org/10.1007/s10891-018-1862-4

[24] Chaubey, V., Nath, G. (2001). Magnetogasdynamic shock waves in a non-ideal gas with radiation heat-flux. J. Nat. Acad. Math. India, 15: 45-57.

[25] Nath, G. (2011). Magnetogasdynamic shock wave generated by a moving piston in a rotational axisymmetric isothermal flow of perfect gas with variable density. Advances in Space Research, 47(9): 1463-1471. https://doi.org/10.1016/j.asr.2010.11.032

[26] Ashraf, S., Ahmad, Z. (1975). Approximate analytic solution of a strong shock with radiation near the surface of the star. Indian Journal of Pure and Applied Mathematics, 6: 1090-1098.

[27] Laumbach, D.D., Probstein, R.F. (1970). Self-similar strong shocks with radiation in a decreasing exponential atmosphere. The Physics of Fluids, 13(5): 1178-1183. https://doi.org/10.1063/1.1693048

[28] Zhuravskaya, T.A., Levin, V.A. (1996). The propagation of converging and diverging shock waves under intense heat exchange conditions. Journal of Applied Mathematics and Mechanics, 60(5): 745-752. https://doi.org/10.1016/S0021-8928(96)00094-9

\section{NOMENCLATURE}

A constant

$a_{\text {sound }}$ speed of sound in non-ideal gas

$a(\mathrm{t}) \quad$ function of $\mathrm{t}$

$a_{\text {isoth }} \quad$ isothermal sound speed

$\mathrm{b} \quad$ van der Waal excluded volume

$\bar{b} \quad$ non-idealness parameter

$\mathrm{b}(\mathrm{t}) \quad$ function of $\mathrm{t}$

$C_{\text {isoth }} \quad$ isothermal compressibility

$C_{v} \quad$ specific heat at constant volume

D non-dimensional density

$E_{\text {Total }}$ total energy of the flow-field behind shock front

$\mathrm{F}$ radiation flux

$\mathrm{f}(\mathrm{t}) \quad$ function of $\mathrm{t}$

$\mathrm{G}$ the gravitational constant

$\mathrm{G}_{0} \quad$ the gravitational parameter 
$\mathrm{m} \quad$ central mass at the origin

$\mathrm{P} \quad$ non-dimensional fluid pressure

$\mathrm{p} \quad$ is the pressure

$\mathrm{R}$ gas constant

$\mathrm{r} \quad$ independent space coordinate

$r_{s} \quad$ Shock radius

$\mathrm{T}$ temperature of the gas

$\mathrm{t}$ independent time coordinate

u fluid velocity

$\mathrm{V}$ non-dimensional fluid velocity

$\mathrm{W}_{\mathrm{s}} \quad$ shock velocity

X similarity variable

\section{Greek letters}

$\rho \quad$ fluid density

$\alpha \quad$ shock velocity variation index $\gamma \quad$ ratio of specific heats

$\beta \quad$ ratio of density across the shock front

$\eta \quad$ arbitrary function of $r$ and $t$

$\lambda$ constant

$\tau \quad$ constant

\section{Subscripts}

a immediately ahead the shock

$\mathrm{n} \quad$ immediately behind the shock

S process of constant entropy

$\mathrm{T} \quad$ process of constant temperature

\section{Superscript}

derivative with respect to $\mathrm{t}$ 